\title{
AN EVOLUTIONARY ALGORITHM FOR UNICAST/ MULTICAST TRAFFIC ENGINEERING
}

\author{
Miguel Rocha, Pedro Sousa \\ Dep. Informatics/ CCTC, Universidade do Minho, Campus de Gualtar, Braga, Portugal \\ mrocha@di.uminho.pt,pns@di.uminho.pt \\ Paulo Cortez \\ Dep. Information Systems/ Algoritmi, Universidade do Minho, Campus de Azurem, Guimaraes, Portugal \\ pcortez@dsi.uminho.pt \\ Miguel Rio \\ Dep. Electric and Electronic Engineering, University College London, Torrington Place, London, U.K. \\ m.rio@ee.ucl.ac.uk
}

Keywords: Traffic engineering, Multicast content, Evolutionary Algorithms, OSPF.

\begin{abstract}
A number of Traffic Engineering (TE) approaches have been recently proposed to improve the performance of network routing protocols, both developed over MPLS and intra-domain protocols such as OSPF. In this work, a TE approach is proposed for routing optimization in scenarios where unicast and multicast demands are simultaneously present. Evolutionary Algorithms are used as the optimization engine with overall network congestion as the objective function. The optimization aim is to reach a set of (near-)optimal weights to configure the OSPF protocol, both in its standard version and also considering the possibility of using multitopology variants. The results show that the proposed optimization approach is able to obtain networks with low congestion, even under scenarios with heavy unicast/multicast demands.
\end{abstract}

\section{INTRODUCTION}

A new plethora of network services is putting strong requirements on TCP/IP networks, for which these were not initially designed. Many of these services will need a multicast enabled network with demanding quality of service (QoS) constraints in the end-to-end data delivery. The advent of 3-play service providers, where the same entity is involved in the network and TV provision is just the first of these scenarios. Interactive TV, virtual reality, videoconferencing, video games or video surveillance are just some of the applications that would gain from QoS enabled multicast content delivery. In these scenarios, data needs to arrive to a set of users with minimal loss (therefore requiring minimization of congestion) and with acceptable end-to-end delays.

Multicast has been present for a while in TCP/IP networks, but its widespread use has never occurred in the Internet. It is, however, used in closed TCP/IP networks where its scalability problems are not a deterrent. In fact, many IPTV and video-on-demand services operate in closed networks using multicast to save bandwidth and enhance QoS levels.
In this context, Traffic Engineering (TE) techniques can be used to improve network performance by achieving near-optimal configurations for routing protocols. TE approaches can be classified into: Multi-Protocol Label Switching (MPLS) (Davie and Rekhter, 2000)(Awduche and Jabbari, 2002) based and pure IP-based intra-domain routing protocols. With MPLS, packets are encapsulated with labels at ingress points, that can be used to route these packets along an explicit label-switched path). Together with resource reservation mechanisms, these capabilities are able to support stringent end-to-end bandwidth guarantees for multicast content delivery. However, the use of MPLS presents significant drawbacks: firstly, it adds significant complexity to the model, since per-flow state has to be stored in every router of the path; secondly, MPLS failure recovery mechanisms are considerably more complex than the typical router convergence ones; finally, it represents a considerable network management overhead.

As regards intra-domain routing protocols, the most commonly used today is Open Shortest Path First(OSPF)(Thomas II, 1998). Here, the administrator assigns weights to each link in the network, 
which are then used to compute the best path from each source to each destination using the Dijkstra algorithm (Dijkstra, 1959). The results are then used to compute the routing tables in each node.

A number of studies have proposed TE procedures which optimize the weights of intra-domain routing protocols to achieve near optimal routing, taking as input the expected traffic demands. This was the approach taken by Fortz and Thorup (2000) where this task was viewed as an optimization problem by defining a cost function that measured network congestion. The authors proved that this task is a NP-hard problem and proposed some local search heuristics that compared well with the MPLS model. Another approach was the use of Evolutionary Algorithms (EAs) to improve these results (Ericsson et al., 2002). Additional research has been carried out with the objective of pursuing multiconstrained QoS optimization, where both traffic demands and delay requirements are considered in the optimization of routing configurations for unicast traffic (Rocha et al., 2006).

In this paper, EAs are employed to reach OSPF weights that optimize network congestion, taking into account both unicast and multicast demands of a given domain. This work is based on the reasoning that in the optimization process both the unicast and multicast demands should be considered simultaneously, in contrast with previous work where optimization is performed in two distinct phases, the first for unicast traffic and the second devoted to multicast optimization (Wang and Pavlou, 2007).

\section{PROBLEM FORMULATION}

\subsection{Unicast Traffic}

In this section, a model for a network only with unicast traffic demands will de described. This is based on the framework proposed in (Fortz and Thorup, 2000). The general routing problem (Ahuja et al., 1993 ) that underpins this work represents routers and links by a set of nodes $(N)$ and $\operatorname{arcs}(A)$ in a directed graph $G=(N, A)$. In this model, $c_{a}$ represents the capacity of each link $a \in A$. A demand matrix $D$ is available, where each element $d_{s t}$ represents the traffic demand between nodes $s$ and $t$. For each $\operatorname{arc} a$, the variable $f_{a}^{(s t)}$ represents how much of the traffic demand between $s$ and $t$ travels over arc $a$. The total unicast load on each arc $a\left(l_{a}\right)$ can be defined as: $l_{a}=\sum_{(s, t) \in N \times N} f_{a}^{s t}$ while the link utilization rate $u_{a}$ is given by: $u_{a}=\frac{l_{a}}{c_{a}}$. It is then possible to define a congestion measure for each link: $\Phi_{a}=p\left(u_{a}\right)$. us- ing a penalty function $p$ that has small values near 0 , but as the values approach the unity it becomes more expensive and exponentially penalizes values above 1 (Fortz and Thorup, 2000).

In OSPF, all arcs have an integer weight, used by each node to calculate the shortest paths to all other nodes in the network, using the Dijkstra algorithm (Dijkstra, 1959). The traffic from a given source to a destination travels along the shortest path. If there are two or more paths with equal length, traffic is evenly divided among the arcs in these paths (load balancing) (Moy, 1998). Let us assume a given a weight assignment, and the corresponding values of $u_{a}$. In this case, the total routing cost is expressed by $\Phi=\sum_{a \in A} \Phi_{a}$ for the loads and penalties $\left(\Phi_{a}\right)$ calculated based on the given OSPF weights. In this way, the OSPF weight setting problem is equivalent to finding the optimal weight value for each link, in order to minimize $\Phi$. The congestion measure can be normalized $\left(\Phi^{*}\right)$ over distinct scenarios to values in the range $[1,5000]$. It is important to note that in the case when all arcs are exactly full $\left(l_{a}=c_{a}\right)$, the value of $\Phi^{*}$ is $10 \frac{2}{3}$, a value that will be considered a threshold that bounds the acceptable working region of the network.

\subsection{Multicast Demands}

A model that considers only multicast traffic in the network will be described, that is based on a the work by Wang and Pavlou (2007). If there are unicast and multicast demands, this model can be used to perform a two-step optimization process (explained in the next section). Consider, as before, a network topology $G=(N, A)$, with arc capacities $\left(c_{a}\right)$. The multicast demands are given for a set of $G$ groups, where for each group $g \in G$ the following parameters are defined: a root node $r_{g}$, a bandwidth demand $M_{g}$ and a a set of receivers $\left(V_{g}\right)$. The multicast optimization problem is typically defined as the computation of a bandwidth constrained Steiner tree, with the objective of minimizing overall bandwidth consumption, using integer programming. The target is to instantiate a number of binary decision variables: $y_{a}^{g}$, are equal to 1 if link $a$ is included in the multicast tree for group $g$; and $x_{a}^{g, k}$ are equal to 1 if link $a$ is included in the multicast tree for group $g$, in the branch from the root node to receiver $k$. The objective function is to minimize the overall bandwidth consumption ( $L 1)$ :

$$
L 1=\sum_{g \in G} \sum_{a \in A} M_{g} \times y_{a}^{g}
$$

The deployment of the obtained Steiner trees can be enforced by using an explicit routing overlay, through MPLS on a per-group basis. An alterna- 
tive with some advantages, previously discussed, is to consider that the routing will be achieved by using an intra-domain protocol such as OSPF. In this case, the tree for a given group will be built from the shortest paths between the root node and each receiver. Therefore, the values assigned to $y_{a}^{g}$ variables will be computed as follows: $y_{a}^{g}$ is equal to 1 if link $a$ is in the shostest path from the root node $g$ to at least one of the receivers in $V_{g}$, and is equal to 0 otherwise.

In previous work (Wang and Pavlou, 2007), EAs have been proposed to optimize OSPF weights for multicast traffic. The objective function used in this case is based on the overall network load (L1) but also on the excessive bandwidth allocated to overloaded links (L2), that can is given by:

$$
\begin{gathered}
L 2=\sum_{a \in A}\left[w_{a} \sum_{g \in G}\left(M_{g} \times y_{a}^{g}\right)-c_{a}\right] \\
w_{a}= \begin{cases}0, & \text { if } \sum_{g \in G} M_{g} \times y_{a}^{g} \leq c_{a} \\
1, & \text { otherwise }\end{cases}
\end{gathered}
$$

The EA's fitness is, therefore, given by:

$$
f(L 1, L 2)=\frac{\mu}{\alpha \times L 1+\beta \times L 2}
$$

where $\mu, \alpha$ and $\beta$ are constants, whose values are set to $10^{7}, 1$ and 10 respectively.

\subsection{Unified Model with Unicast and Multicast Demands}

In this work, a unified approach will be proposed that is able to reach OSPF weights that optimize the network congestion measure, simultaneously considering unicast and multicast demands. In this case, the multicast load for a given link $a$ can be computed as: $m l_{a}=\sum_{g \in G} M_{g} \times y_{a}^{g}$. The values of $y_{a}^{g}$ will be calculated from the OSPF weights as explained in the previous section. So, the total load on a given arc $a$ is given by: $l_{a}=m l_{a}+u l_{a}$, where $u l_{a}$ is the unicast load in $\operatorname{arc} a$ (given by $l_{a}$ in the previous section). It should noted that $l_{a}$ here takes the meaning of the total load in the network, while in Section $2.1 l_{a}$ is only used for unicast loads since in that case those were the only loads considered. After calculating the overall values of $l_{a}$ for all links, the process proceeds as described in Section 2.1, in order to reach the normalized congestion measure $\Phi^{*}$.

Another interesting measure of the network performance in this scenario is the excessive bandwidth in overloaded links (BOL). This is a generalization of L2 but now applied to the global loads and not only to the multicast traffic. This is defined as:

$$
\begin{gathered}
B O L=\sum_{a \in A} z_{a}\left(l_{a}-c_{a}\right) \\
z_{a}= \begin{cases}0, & \text { if } l_{a} \leq c_{a} \\
1, & \text { otherwise }\end{cases}
\end{gathered}
$$

\section{OPTIMIZATION ALGORITHMS}

\subsection{Evolutionary Algorithms}

Evolutionary Algorithms (EAs) (Michalewicz, 1996) are a popular family of optimization methods, inspired in the biological evolution. These methods work by evolving a population, i.e. a set of individuals, each encoding solutions to a target problem in an artificial chromosome. Each individual is evaluated through a fitness function, that assigns it a numerical value, corresponding to the quality of the encoded solution. EAs are stochastic methods due to their selection process. In fact, individuals selected to create new solutions are taken from the population using probabilities. Highly fit individuals have a higher probability of being selected, but the less fit still have their chance.

In the proposed EA, each individual encodes a solution in a direct way, i.e. as a vector of integer values, where each value corresponds to the weight of an arc in the network (the values range from 1 to $w_{\max }$ ). Therefore, the size of the individual equals the number of links in the network. If multiple topologies are used, i.e. different sets of weights for unicast and multicast, the size of the individual is twice the number of links and the two sets of weights are encoded linearly, i.e. the first $L$ genes encode the weights for unicast traffic, while the latter $L$ links encode the weights for multicast ( $L$ is the number of links).

The weight values for individuals in the initial population are randomly generated, taken from a uniform distribution. In order to create new solutions, several reproduction operators were used, more specifically two mutation and one crossover operator:

- Random Mutation, replaces a given weight value by a random value, within the allowed range;

- Incremental/decremental Mutation, replaces a given weight value $w$ by $w+1$ or by $w-1$ (with equal probabilities);

- Uniform crossover, a standard crossover operator (Michalewicz, 1996).

The operators are all used to create new solutions with equal probabilities. The selection procedure is done by converting the fitness value into a linear ranking in the population, and then applying a roulette 
wheel scheme. In each generation, $50 \%$ of the individuals are selected from the previous generation, and $50 \%$ are bred by the application of the genetic operators over selected parents. A population size of 100 individuals was considered.

\subsection{Optimization Approaches}

Three distinct optimization approaches are compared, with the aim to optimize OSPF weights in networks where both unicast and multicast demands are available. All these methods use EAs as the optimization engine. The first method is a 2-step optimization process (2S), based on the proposal from Section 2.2 (Wang and Pavlou, 2007), that can be described as:

1. the OSPF weights are optimized (using EAs) to minimize congestion penalties $\left(\Phi^{*}\right)$ only taking into account the unicast demands;

2. the bandwidths used for each link in unicast traffic are deduced from the link capacities;

3. a second optimization process is conducted, where a different set of weights is calculated from multicast traffic only, by running a new EA with $\mathrm{f}(\mathrm{L} 1, \mathrm{~L} 2)$ (Equation 4) as the fitness function.

This method assumes that a protocol that allows multiple sets of weights, each for a distinct type of traffic, is deployed. This is the case, for instance, of the multi-topology protocol MT-OSPF (Psenak et al., 2006). The remaining alternatives are based on the model proposed in Section 2.3. Using this model, two different optimization approaches may be followed:

- Single topology (ST), i.e. a single set of OSPF weights is used for both types of traffic demands;

- Multiple topologies (MT), i.e. two sets of OSPF weights are used, one for unicast traffic and the other for multicast demands. In this case, as before, a multi-topology protocol has to be used.

\section{EXPERIMENTS AND RESULTS}

\subsection{Experimental Setup}

To evaluate the proposed algorithms, a number of experiments were conducted. The experimental platform used in this work is presented in Figure 1. All algorithms and the OSPF routing simulator were implemented using the Java language. A set of 3 network topologies was created using the Brite topology generator (Medina et al., 2001), varying the number of nodes $(N=30,50,80)$ and the average degree of each node was kept in $(m=4)$. This resulted in networks ranging with 110, 190 and 310 links, respectively.

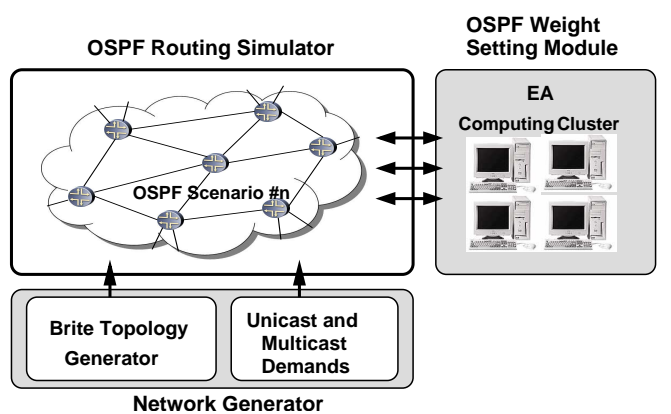

Figure 1: Experimental platform for EA's performance evaluation.

The link bandwidth (capacity) was generated by a uniform distribution between 1 and 10 Gbits/s. The networks were generated using the Barabasi-Albert model, using a heavy-tail distribution and an incremental grow type (parameters $H S$ and $L S$ were set to 1000 and 100). Next, the unicast demand matrices $(D)$ were generated (two distinct matrices for each network). A parameter $\left(D_{p}\right)$ was considered, representing the expected mean of congestion in each link (values for $D_{p}$ were 0.2 and 0.3 ).

The generation of the multicast traffic demands was based on the following: Firstly, for each network the number of groups $G$ was set equal to the number of nodes. The root node for each group was randomly chosen from the set of nodes (with equal probabilities). For each group, the number of receivers was generated from the range $[2, n / 2]$, where $n$ is the number of nodes. The set of receivers $V_{g}$ was created with the given cardinality, by randomly selecting a set of nodes different from the root. Finally, the demand $M_{g}$ was generated taking a parameter $(R)$ into account. $R$ is defined as the ratio between the total multicast demands and the total unicast demands. Given $R$ and given the unicast demands, a target is calculated for the total multicast demands. The group demands are generated by dividing the target value by the different groups in an uneven way, so that groups with different demands are created resulting in a more plausible scenario. By using $R$, a better understanding of the results is possible since an approximate idea of the trade-offs between unicast and multicast is known. The values of $R$ used were 1 and 0.5 .

The termination criteria for all optimization approaches consisted in a maximum number of solutions evaluated. This value ranged from 100000 to 300000 , increasing linearly with the number of links. For all cases, $w_{\max }$ was set to 20 and 20 runs were executed and the results presented are the means. 
Table 1: Results for the network with 30 nodes.

\begin{tabular}{l|l|ccc}
\hline Demands & Metric & ST & MT & $2 \mathrm{~S}$ \\
\hline $\mathrm{D}=0.2$ & $\Phi^{*}$ & 1.38 & 1.31 & 1.83 \\
$\mathrm{R}=0.5$ & BOL & 0 & 0 & 42 \\
& $\mathrm{~L} 1\left(\times 10^{5}\right)$ & 1.10 & 1.09 & 0.96 \\
\hline $\mathrm{D}=0.2$ & $\Phi^{*}$ & 3.27 & 3.00 & 8.66 \\
$\mathrm{R}=1.0$ & $\mathrm{BOL}$ & 160 & 156 & 1138 \\
& $\mathrm{~L} 1\left(\times 10^{5}\right)$ & 2.11 & 2.11 & 1.97 \\
\hline $\mathrm{D}=0.3$ & $\Phi^{*}$ & 3.32 & 2.83 & 7.78 \\
$\mathrm{R}=0.5$ & $\mathrm{BOL}$ & 257 & 128 & 875 \\
& $\mathrm{~L} 1\left(\times 10^{5}\right)$ & 1.50 & 1.54 & 1.39 \\
\hline $\mathrm{D}=0.3$ & $\Phi^{*}$ & 66.2 & 38.0 & 93.5 \\
$\mathrm{R}=1.0$ & $\mathrm{BOL}$ & 14239 & 7040 & 16820 \\
& $\mathrm{~L} 1\left(\times 10^{5}\right)$ & 3.05 & 3.04 & 2.84 \\
\hline
\end{tabular}

Table 2: Results for the network with 50 nodes.

\begin{tabular}{l|l|ccc}
\hline Demands & Metric & ST & MT & $2 \mathrm{~S}$ \\
\hline $\mathrm{D}=0.2$ & $\Phi^{*}$ & 1.38 & 1.34 & 1.90 \\
$\mathrm{R}=0.5$ & BOL & 0 & 0 & 56 \\
& L1 $\left(\times 10^{5}\right)$ & 2.10 & 2.11 & 1.91 \\
\hline $\mathrm{D}=0.2$ & $\Phi^{*}$ & 2.29 & 1.85 & 5.69 \\
$\mathrm{R}=1.0$ & BOL & 99 & 9 & 1166 \\
& L1 $\left(\times 10^{5}\right)$ & 4.50 & 4.54 & 4.18 \\
\hline $\mathrm{D}=0.3$ & $\Phi^{*}$ & 2.44 & 2.04 & 3.15 \\
$\mathrm{R}=0.5$ & BOL & 88 & 30 & 241 \\
& L1 $\left(\times 10^{5}\right)$ & 2.58 & 2.63 & 2.38 \\
\hline $\mathrm{D}=0.3$ & $\Phi^{*}$ & 21.9 & 7.47 & 26.8 \\
$\mathrm{R}=1.0$ & BOL & 11820 & 2862 & 10880 \\
& L1 $\left(\times 10^{5}\right)$ & 5.58 & 5.72 & 5.39 \\
\hline
\end{tabular}

\subsection{Results}

In Tables 1, 2 and 3 the results for the optimization approaches are shown. In the first column, the demand generation parameters $(D, R)$ are shown. The second column shows the metrics, while columns 3,4 and 5 show the results of each optimization approach, according to the metrics. Four scenarios are given for each network: the first rows show the example with less demands, while the last rows show the worst case scenario. The middle rows show two intermediate scenarios, where in one case the unicast demands are low, but the multicast demands are high and in the next the reverse takes place.

A different perspective is shown in Figures 2, 3 and 4 , where the congestion measure $\left(\Phi^{*}\right)$ for the three networks is plotted. In each plot, the four scenarios in terms of demands are shown. The values are shown in a logarithmic scale, given the exponential nature of the penalty function.
Table 3: Results for the network with 80 nodes.

\begin{tabular}{l|l|ccc}
\hline Demands & Metric & ST & MT & 2S \\
\hline $\mathrm{D}=0.2$ & $\Phi^{*}$ & 1.44 & 1.38 & 1.74 \\
$\mathrm{R}=0.5$ & BOL & 0 & 0 & 7 \\
& L1 $\left(\times 10^{5}\right)$ & 2.54 & 2.63 & 2.33 \\
\hline $\mathrm{D}=0.2$ & $\Phi^{*}$ & 2.09 & 1.90 & 3.02 \\
$\mathrm{R}=1.0$ & BOL & 160 & 77 & 582 \\
& L1 $\left(\times 10^{5}\right)$ & 5.01 & 5.12 & 4.64 \\
\hline $\mathrm{D}=0.3$ & $\Phi^{*}$ & 2.63 & 2.50 & 3.97 \\
$\mathrm{R}=0.5$ & BOL & 227 & 247 & 821 \\
& L1 $\left(\times 10^{5}\right)$ & 3.48 & 3.59 & 3.18 \\
\hline $\mathrm{D}=0.3$ & $\Phi^{*}$ & 17.7 & 10.4 & 32.6 \\
$\mathrm{R}=1.0$ & $\mathrm{BOL}$ & 11426 & 6856 & 15242 \\
& $\mathrm{~L} 1\left(\times 10^{5}\right)$ & 7.25 & 7.49 & 6.90 \\
\hline
\end{tabular}

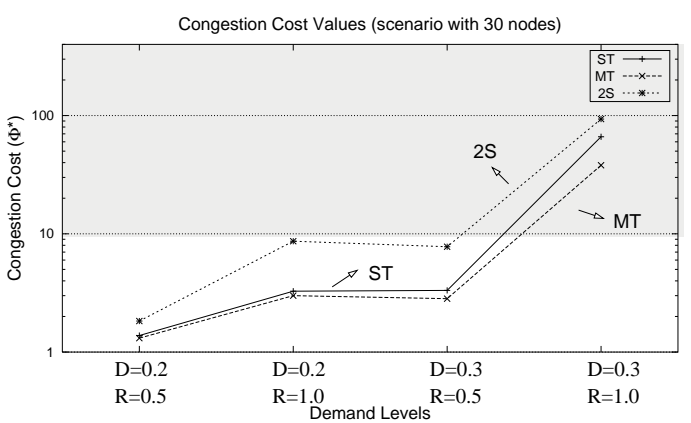

Figure 2: A plot of the results for congestion measure (network with 30 nodes).

\subsection{Discussion}

The first conclusion to draw from these results is that $2 \mathrm{~S}$ leads to sub-optimal results, in terms of overloaded links and network congestion (visible both in the BOL and $\Phi^{*}$ ). Both the MT and ST show better results, being able to keep the network in an acceptable behaviour in most scenarios. The scenario shown in the last rows $(D=0.2$ and $R=1.0)$ is an extreme case, where the demands are quite high. Although this would not be acceptable in a real world network, it is useful to have an idea of how the distinct optimization methods scale. When comparing ST and MT, the results are quite near in the low demand scenarios. In these cases, the gain obtained by using MT is not impressive. The gap increases with the values of $D$ and $R$, i.e. as the problem gets harder. In practical terms, this would mean that if the network has lots of resources in terms of bandwidth and low demands, it is probably not worth to pay the cost of deploying a multi-topology protocol. On the other hand, using this kind of protocol allows the network to support higher demands with the same bandwidth resources.

Regarding the L1 values, the best alternative is 


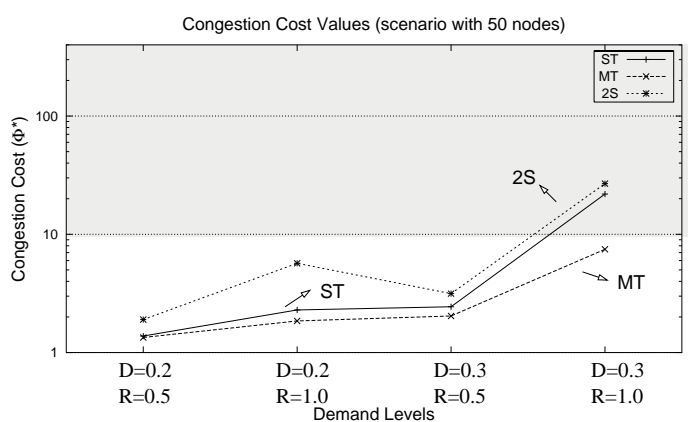

Figure 3: A plot of the results for congestion measure (network with 50 nodes.

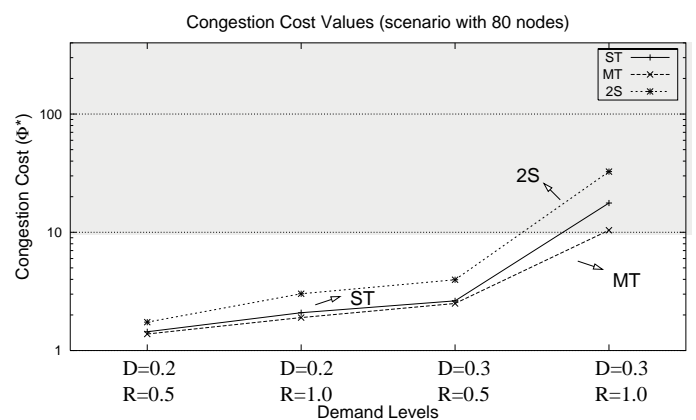

Figure 4: A plot of the results for congestion measure (network with 80 nodes.

$2 \mathrm{~S}$ and thus this method allocates better the multicast traffic. This is not surprising, since L1 is part of the objective function in this case. However, this solution does not result in a good performance when the network congestion is taken as a whole. So, this solution would lead to high levels of loss, that would be unacceptable in most cases.

\section{CONCLUSIONS}

The optimization of OSPF weights brings important tools for traffic engineering, without demanding modifications on the basic network model. This work presented EAs for routing optimization in networks with unicast and multicast demands. Resorting to a set of network configurations and unicast/ multicast demands, it was shown that the proposed EAs were able to provide OSPF weights that can lead to good network behaviour. The proposed approach was favourably compared to a 2-step optimization procedure, proposed in previous work, that leads to suboptimal results in terms of network congestion and overloaded links. The advantages of using a multitopology protocol in these scenarios were also studied and it was concluded that these are most advantageous when the network bandwidth resources are limited.
The main contribution of this work is the capability of optimizing the OSPF weights considering all factors involved (i.e. all types of traffic). Using the proposed methods, the network administrator can decide if a multi-topology protocol is needed or simply use a standard implementation of OSPF. In the future, we will proceed with the integration of further QoS constraints in the model, with a priority on the introduction of end-to-end delays.

\section{ACKNOWLEDGEMENTS}

This work was supported by the Portuguese Foundation for Science and Technology under project POSC/EIA/59899/2004, partially funded by FEDER.

\section{REFERENCES}

Ahuja, R., Magnati, T., and Orlin, J. (1993). Network Flows. Prentice Hall.

Awduche, D. and Jabbari, B. (2002). Internet traffic engineering using multi-protocol label switching (MPLS). Computer Networks, 40:111-129.

Davie, B. and Rekhter, Y. (2000). MPLS: Multiprotocol Label Switching Technology and Applications. Morgan Kaufmann, USA.

Dijkstra, E. (1959). A note on two problems in connexion with graphs. Numerische Mathematik, 1(269-271).

Ericsson, M., Resende, M., and Pardalos, P. (2002). A Genetic Algorithm for the Weight Setting Problem in OSPF Routing. J. of Combinatorial Optimization, 6:299-333.

Fortz, B. and Thorup, M. (2000). Internet Traffic Engineering by Optimizing OSPF Weights. In Proceedings of IEEE INFOCOM, pages 519-528.

Medina, A., Lakhina, A., Matta, I., and Byers, J. (2001). BRITE: Universal Topology Generation from a User's Perspective. Technical Report 2001-003.

Michalewicz, Z. (1996). Genetic Algorithms + Data Structures $=$ Evolution Programs. Springer-Verlag, USA, third edition.

Moy, J. (1998). OSPF, Anatomy of an Internet Routing Protocol. Addison Wesley.

Psenak, P., Mirtorabi, S., Roy, A., Nguyen, L., and PillayEsnault, P. (2006). Multi-topology (mt) routing in ospf (internet draft).

Rocha, M., Sousa, P., Rio, M., and Cortez, P. (2006). Qos constrained internet routing with evolutionary algorithms. In Proc. IEEE Conference Evolutionary Computation, pages 9270-9277. IEEE Press.

Thomas II, T. (1998). OSPF Network Design Solutions. Cisco Press.

Wang, N. and Pavlou, G. (2007). Traffic Engineered Multicast Content Delivery Without MPLS Overlay. IEEE Transactions on Multimedia, 9(3). 